\title{
Variable quotas, irreversible investment and optimal capacity in the fisheries
}

\author{
SJUR D. FLÅM† \\ Keywords: Fisheries economics, Control theory, Stochastic optimization
}

We study the adaptation of a fishing fleet to a situation involving variable quotas of catch and irreversible investment. The theoretical results obtained here are applied to Norwegian industrial fisheries.

\section{Introduction}

The domain of fisheries management is beset with uncertain factors, many of which are associated with the population dynamics of commercial stocks. Notably the recruitment to the parent stock is highly variable and not easy to predict. As a result, annual quotas of the allowable catch will fluctuate so that even a potential equilibrium will take the form of a probabilistic distribution. In fact, the concept of a stochastic steady state means that all random parameters are obtained by repetitive sampling from a time-invariant distribution (Arrow, Bekermann and Karlin 1958).

This paper is concerned with how to adapt the catch capacity to such an equilibrium situation. Here the quotas of catch are random and unveiled each year as drawings from a permanent lottery.

This emphasis on a situation where the fish stock is in stochastic equilibrium is certainly worthy of criticism. The only defence is our hunch that an attack on more complex problems will be facilitated by a thorough understanding of simple models like the one studied here.

Under this assumption, that the stock is in equilibrium, we shall study the best path of capacity expansion as well as the problem of how to retract if excess capacity is in store.

These issues reside in the domain of capital management (Mirman and Spulber 1982, Mirman and Zilcha 1975, or Reed 1974) and have been studied in a context akin to ours by several authors (Charles 1981 or Clark et al. 1979). However, our focus is as much on the comparative statics of the equilibrium as it is on capital adjustment.

A primary aim is to explore how the economic parameters and the variability of the annual quotas work together to affect the optimal long-term choice of catch capacity. In this endeavour we are motivated by several questions:

- If investment is irreversible, how does the path of capital expansion differ from that of retraction?

-When is it prudent to ignore stochastic considerations?

- What are the effects of misspecifying a stochastic model?

- How will greater uncertainty affect the choice of capacity?

Received 23 December 1985

† CMI, Dept. of Science and Technology, N-5036 Fantoft, Bergen, Norway 


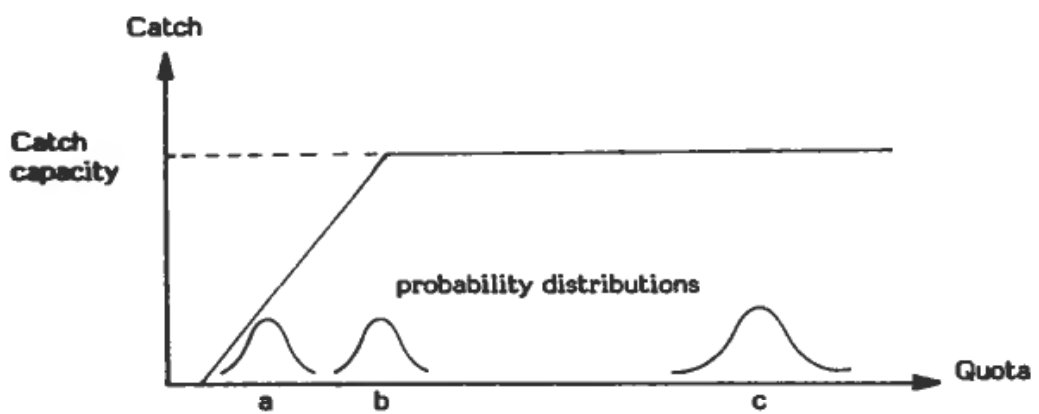

Figure 1. The relation between catch and quota for given capacity.

In addressing these questions we shall deliberately steer away from much of the complexity of the real world and concentrate on the following principal mechanism.

Suppose that planning is undertaken with no risk aversion. This means that the utility function of money is linear and the relevant criterion for choosing between lotteries is the expected present value of the associated flow of income. Moreover, suppose that costs and revenues are linear functions of the intensity of productive activities. Then it seems that the entire decision problem is linear throughout, and consequently, uncertainty appears to be of no importance at all. This is only on the surface of things, however. It takes only a moment's reflection to acknowledge that catch capacity might occasionally be constraining, and, most important, this introduces an important nonlinearity into the problem. This is illustrated in Figure 1 above.

In Figure 1 note that if the quota allotted to the fleet is distributed as alternative a or c, then uncertainty has no bearing in terms of expected catch. By contrast, if uncertainty occurs in the region of 'strict concavity' (the kink point), as does alternative $b$, then it becomes mandatory to account for the resulting lack of symmetry, namely, no addition to the quota above level b may compensate for the loss incurred if the quota drops below this level. The upshot of all this is that even under risk neutrality in terms of money income, the capacity constraint works its way through the problem so as to render us risk averse with respect to variations in the quota.

This paper explores how we ought to adapt to this kind of situation. Right at the outset one should be aware of the dynamic feature of this planning problem. In fact, it embodies two-stage optimization. In the first stage one chooses the desired level of catch capacity. Then, in the second stage, being faced with a specific realization of the quota, one may have to mitigate adverse effects of a tight capacity constraint. In this respect there are several avenues to pursue. For example, one may, if need be, extend the fishing season, increase the speed of the vessels, work overtime at the processing factories, trade quotas and so on, cf. (Brekke and Wallace 1984 or Flam 1984). Here we shall simply ignore all of these possibilities and assume that the only feasible second-stage action is to catch as much as possible of the quota in the usual manner of operation. Admittedly this simplification misrepresents the flexibility of the system, that is, the actual ability to cope with random variations is underestimated. Therefore we shall end up providing one-sided estimates of the optimal catch capacity, i.e. we shall systematically produce overestimates.

Yet we think that the simple approach adopted here has its own merits. In the first place it easily provides approximations to the optimal capacity. Secondly, these 
approximations have a built-in safety margin because second-stage recourse actions have been neglected. Such actions would, if needed, occasionally increase the capacity to the effect of producing a higher long-term profit.

The paper is organized as follows. Section 2 introduces the model of optimal investment under conditions of irreversibility. The best investment strategy is described in Section 3, theorems 1 and 2. It will be seen that the irreversibility of investment induces a glaring lack of symmetry in the path of capital adjustment. Specifically, if there is an initial shortage of capacity, then an immediate expansion up to the long-term level is justified. By contrast, if capacity is in excess supply, then only some part of it should potentially be sold (or scrapped) at once. Afterwards the long-term target level is to be approached only by the attractive force of depreciation.

We then, in Section 4, continue to investigate how this target level, the golden equilibrium, depends on the given data. Finally Section 5 sets the obtained results to work on a practical application. The example discussed there is the capelin fisheries in the Barents Sea. We demonstrate that existing capacity should be reduced, and we also suggest how the path of capacity retraction should look.

\section{The model}

This section introduces the elements of the decision problem. The overall objective is to maximize the present value of the flow of profits. In order to state the specific form of this criterion, we first need to specify the dynamics and the economics of the problem.

\section{Dynamics}

Denote by $Q_{t}$ the catch capacity of the fleet at the very beginning of year $t$. This capacity is immediately changed to $Q_{t}+I_{t}$ as a result of investing $I_{t} \geqslant-Q_{t}$. The capacity in the subsequent year is given by

$$
Q_{t+1}=\beta \times\left(Q_{t}+I_{t}\right)
$$

where the initial capacity $Q_{0} \geqslant 0$ is specified at the outset and $\beta \in(0,1)$ is a factor representing annual depreciation.

The sequence of catches (i.e. the quotas) $q_{0}, q_{1}, q_{2} \ldots$ is assumed to be a realization of a stochastic process with independent, identically distributed elements. This assumption is a stringent one and requires some justification. Towards this we discuss here a particular model of fishery management which is adapted to the example that follows in Section 5. Consider a commercial fishery exploiting only the mature fraction of a stock that migrates to spawn. After spawning the parent stock dies. Suppose that the operating principle of the managment is to allow for a constant escapement of fish to spawn in order to secure a stable recruitment to the stock. If this regulatory scheme is effective, and the environment supporting the stock is stable in a probabilistic sense, then it is realistic to conceive of the annual quotas as a stochastic residual being sampled from a time-invariant distribution (Flam 1981). In fact, we shall do so. We regard the probability distribution of the quotas as data of the problem. ${ }^{1}$ We now turn to the economic parameters of the problem.

1 The example above points to the fact that the distribution itself will be affected by the regulatory scheme. Thus the choice of this distribution is also a task for constrained optimization. 


\section{Economics}

Let $\pi\left(Q_{t}+I_{t}, q_{t}\right)$ denote the single-period profit net of investment costs. This profit may arise by implementing, in the short term, the most effective activity to catch as much as possible of the quota $q_{t}$ given the capacity $Q_{t}+1_{t}$. By way of example, $\pi\left(Q_{t}+I_{t}, q_{t}\right)$ might equal the optimal value of activity analysis stated and solved as a linear programming problem (Fläm and Storøy 1982). We shall invariably assume that $\pi$ is concave in the first argument.

It remains to account for the $\operatorname{cost} c\left(I_{t}\right)$ of investment. Note that we allow for both proper investment $\left(I_{t} \geqslant 0\right)$ and disinvestment $\left(0 \geqslant I_{t} \geqslant-Q_{t}\right)$. The strategic response to fluctuations in the quota is likely to be constrained by irreversibility of capital and costs of adjustment. These inflexibilities imply that the current level of capacity will often represent a commitment to a particular strategy.

We shall represent the complications of irreversible investment in a simplified but still realistic manner, namely, let

$$
c\left(I_{t}\right)=\left\{\begin{array}{lll}
c^{+} I_{t} & \text { if } & I_{t}>0 \\
c^{-} I_{t} & \text { if } & I_{t} \leqslant 0
\end{array}\right.
$$

where $0 \leqslant c^{-} \leqslant c^{+}$.

The pathological case $c^{-}>c^{+}$is excluded from further consideration because in this case one would become infinitely rich just buying and selling capacity. Note that $c^{-} \leqslant c^{+}$implies that $c$ is a convex function. The important specification $c^{-}<c^{+}$represents the case where capital is non-malleable (Clark, Clarke and Munro 1979). Generally this case is represented by $c(\cdot)$ being strictly convex at zero. This happens if the productive equipment is highly specialized and cannot be fully utilized in any alternative activity.

\section{The criterion}

We now collect terms with the objective to maximize the expected present value of the flow of pure profit, that is, we want to solve problem

$$
(P) \text { : maximize } E \sum_{t=0}^{\infty} \alpha^{t}\left\{\pi\left(Q_{t}+I_{t}, q_{t}\right)-c\left(I_{t}\right)\right\}
$$

with respect to $I_{0}, I_{1}, \ldots$ subject to $Q_{t+1}=\beta \times\left(Q_{t}+I_{t}\right)$ with $Q_{0}$ given and $\alpha \in(0,1)$ being a discount factor.

In the next section we shall give the general form of the solution of problem $(P)$ the focus being there on the best path of capacity adjustment.

\section{Capacity adjustment}

\subsection{Introduction}

The aim of this section is to characterize the solution of $(P)$. We shall be concerned with the transient part of the optimal path and also identify the golden equilibrium. It will be seen that the case of capacity retraction differs significantly from that of expansion. In the latter case an immediate adjustment to the equilibrium level is optimal, whereas if capacity is excessive, the best choice has less of a bang-bang nature. In fact, capacity should be reduced from very high levels with no 
delay, only to a certain level above the golden equilibrium. From this level we approach the equilibrium by the force of depreciation.

In particular, it will be seen that the optimal strategy is myopic in nature. This feature seems to hinge on the linearity of adjustment cost, see capital adjustment models in (Arrow, Bekermann and Karlin 1958) and inventory models in (Heyman and Sobel 1984). We find it interesting to note that 'one-sided linearity' in the function $c(\cdot)$ suffices to preserve the myopic nature of the solution. This point has not been fully made in the theory of sequential decisions.

\subsection{Analysis}

Introduce $\pi(Q):=E \pi(Q, q), x_{t}:=Q_{t}+I_{t}$, and note that $I_{t}=x_{t}-\beta x_{t-1}$ for $t \geqslant 1$. Then (2) becomes

$$
\pi\left(Q_{0}+I_{0}\right)-c\left(I_{0}\right)+\sum_{t=1}^{\infty} \alpha^{t}\left\{\pi\left(x_{t}\right)-c\left(x_{t}-\beta x_{t-1}\right)\right\}
$$

We shall find it instructive first to identify the optimal strategy in a heuristic manner. Later on optimality will be demonstrated by more formal arguments. We start with discussing the case where the initial capacity $Q_{0}$ is very small. Then it seems reasonable to assume that investment must be undertaken in each and every period, i.e. $I_{t} \geqslant 0$ for all $t \geqslant 0$. Under this assumption (3) reads

$$
\pi\left(x_{0}\right)-c^{+} I_{0}+\alpha \beta c^{+} x_{0}+\sum_{t=1}^{\infty} \alpha^{t}\left[\pi\left(x_{t}\right)-(1-\alpha \beta) c^{+} x_{t}\right]
$$

Note that (4) is completely decomposed with respect to time. Thus we may and should optimize in each single period without any concern for dynamic effects. In order to maximize (4) introduce

$$
P(x):=\pi(x)-(1-\alpha \beta) c^{+} x
$$

Note that $P$ is a concave function. We shall safely assume that $P(0)=0, P(x)>0$ for some $x>0$, and $P(\infty)=-\infty$.

Recall that $\pi(Q, q)$ is supposed to be concave in the first variable. Then $\pi(Q)$ is also concave, and the one-sided derivatives $\pi_{+}^{\prime}(Q), \pi_{-}^{\prime}(Q)$ exist for all $Q>0$ with

$$
\pi_{-}^{\prime}(Q) \geqslant \pi_{+}^{\prime}(Q)
$$

Denote the interval $\left[\pi_{+}^{\prime}(Q), \pi_{-}^{\prime}(Q)\right]$ by $\partial \pi(Q)$. In the terminology of convex analysis $\partial \pi(Q)$ is the set of supergradients of $\pi$ at $Q$. In general, the assumption that $\pi(Q)$ is differentiable is not quite realistic. This motivates the introduction of $\partial \pi(Q)$.

The standard Fermat's rule of setting the derivative equal to zero, yields that $Q^{*}$ maximizes (4) if and only if

$$
(1-\alpha \beta) c^{+} \in \partial \pi\left(Q^{*}\right)
$$

In the special case where $\pi$ is differentiable at $Q^{*}$, the optimality condition (6) would read

$$
(1-\alpha \beta) c^{+}=\pi^{\prime}\left(Q^{*}\right)
$$

Later on we shall see that (6) characterizes a long-term equilibrium. In fact, it is optimal eventually to reach a $Q^{*}$ solving (6) irrespective of the starting point $Q$. We therefore pause to comment briefly upon the meaning of (6).

In equilibrium the left-hand side of (6) equals marginal cost of increasing capac- 
ity in one single period. By temporarily expanding capacity, we incur an immediate outlay $c^{+}$per unit and we are relieved of the expense $\beta c^{+}$associated with replacement one period ahead. (6) says that the resulting marginal cost 'equals' marginal revenues.

We also emphasize that the golden equilibrium is totally unaffected by the parameter $c^{-}$as intuition would indicate. Namely, in equilibrium only replacement with new equipment will take place.

We now summarize our findings so far: If $I_{t} \geqslant 0$ is optimal for all $t \geqslant 0$, then (6) is both necessary and sufficient for optimality. This result is not quite satisfying since it is conditional upon $I_{t} \geqslant 0$ for all $t \geqslant 0$. In the following theorem we partly mitigate this situation by taking a first step towards a full identification of the optimal strategy.

THEOREM 1. Suppose $Q_{0} \leqslant Q^{*}$ where $Q^{*}$ solves the inclusion (the generalized equation)

$$
(1-\alpha \beta) c^{+} \in \partial \pi\left(Q^{*}\right)
$$

Then it is optimal to invest up to the level $Q^{*}$ immediately and to maintain capacity at this level forever after.

Proof

(i) The proof is in two steps. We first identify a sufficient condition for optimality and then verify that the proposed strategy is indeed optimal. It is convenient to introduce

$$
u\left(x_{t-1}, x_{t}\right):=\pi\left(x_{t}\right)-c\left(x_{t}-\beta x_{t-1}\right)
$$

with $x_{-1}=\beta^{-1} Q_{0}$ and $x_{t}=Q_{t}+I_{t}$. Then (3) becomes

$$
\sum_{t=0}^{\infty} \alpha^{t} u\left(x_{t-1}, x_{t}\right)
$$

Suppose for some sequences $x_{t}, p_{t}, t \geqslant-1$, we have

$$
\left(p_{t-1},-\alpha p_{t}\right) \in \partial u\left(x_{t-1}, x_{t}\right)
$$

for all $t \geqslant 0$.

Clearly, we may assume that any such sequence $\left(x_{t}\right)_{t=1}^{\infty}$ is bounded. It follows that $\left(p_{t}\right)_{t=-1}^{\infty}$ is also bounded. Then we claim that the trajectory $x_{t}, t \geqslant$ -1 is optimal. In order to see this, consider any feasible bounded trajectory $y_{t}, t \geqslant-1$ starting at $y_{-1}=x_{-1}$. Then by the concavity of $u$,

$$
u\left(y_{t-1}, y_{t}\right) \leqslant u\left(x_{t-1}, x_{t}\right)+p_{t-1}\left(y_{t-1}-x_{t-1}\right)-\alpha p_{t}\left(y_{t}-x_{t}\right)
$$

Multiply this last equation by $\alpha^{t}$ and sum from $t=0$ to $t=T$ to obtain

$$
\sum_{t=0}^{T} \alpha^{t} u\left(x_{t-1}, x_{t}\right) \geqslant \sum_{t=0}^{T} \alpha^{t} u\left(y_{t-1}, y_{t}\right)+\alpha^{T+1} p_{T}\left(y_{T}-x_{T}\right)
$$

Since $\alpha^{T} p_{T}\left(y_{T}-x_{T}\right) \rightarrow 0$, we let $T$ tend to infinity and conclude that $x_{t}, t \geqslant-1$ is indeed optimal.

(ii) Now consider the trajectory

$$
x_{-1}=\beta^{-1} Q_{0}, x_{t}=Q^{*} \text { for all } t \geqslant 0 .
$$


Observe that in general

$$
\partial u\left(x_{t-1}, x_{t}\right)=\left(\beta \partial c\left(x_{t}-\beta x_{t-1}\right), \partial \pi\left(x_{t}\right)-\partial c\left(x_{t}-\beta x_{t-1}\right)\right)
$$

Then $\partial u\left(Q^{*}, Q^{*}\right)=\left(\beta c^{+}, \partial \pi\left(Q^{*}\right)-c^{+}\right)$because $Q^{*}>0$. Thus, it suffices by the results of (i), namely the inclusion $(*)$, to show that

$$
-\alpha \beta c^{+} \in \partial \pi\left(Q^{*}\right)-c^{+}
$$

but this is the same inclusion as (6). This completes the proof. Q.E.D.

We then turn to the more intricate question: What is the best action if $Q_{0}>Q^{*}$ ? Again we shall be guided by economic heuristics.

Trivially if $c^{-}=0$, all of excess capacity should be kept in store to depreciate until it crosses the level $Q^{*}$. By contrast, if $c^{-}>0$, then there are some holding costs implicitly associated with excess capaciy, namely the opportunity cost of capital. If the initial holding of capacity is very high, this opportunity cost will dominate over the benefits furnished by capacity with low marginal productivity.

Thus it seems that an optimal trajectory should start by selling capacity, if any at all. Thereafter we embark on an interim path of depreciation until we cross the level $Q^{*}$ for the purpose of settling there permanently. We now intend to formalize this argument and state it more precisely. Thereafter comes the best time to prove that this strategy is in fact optimal.

We shall need some notation. Let

$$
T(Q)=\min \left\{t: \beta^{t} Q<Q^{*}\right\}
$$

be the minimal time needed to cross the level $Q^{*}$ defined by (6). By theorem 1 , if ever $Q_{t} \leqslant Q^{*}$, then it is optimal to restore the level $Q^{*}$ immediately and stay put. Thus at time $T(Q)$ we should invest the amount

$$
r(Q):=Q^{*}-\beta^{T(Q)} Q
$$

at the unit cost $c^{+}$. Observe that $T(Q)$ is an increasing step function which is differentiable everywhere from the right. Thus

$$
r_{+}^{\prime}(Q)=-\beta^{T(Q)}
$$

Consider two alternative actions at the level $Q+\Delta Q$ : Either sell the amount $\Delta Q>0$ immediately to obtain the value $c^{-} \Delta Q$, or keep it. Suppose $\Delta Q$ is so small that $T(Q)=T(Q+\Delta Q)$. Recall that no replacement takes place before time $T(Q)$. Then the difference between these two projects is

$$
\sum_{t=0}^{T(Q)-1} \alpha^{t}\left[\pi\left(\beta^{t}(Q+\Delta Q)\right)-\pi\left(\beta^{t} Q\right)\right]-\alpha^{T(Q)}[r(Q+\Delta Q)-r(Q)] c^{+}-c^{-} \Delta Q
$$

Divide (i) by $\Delta Q$ and let $\Delta Q \downarrow 0$.

Then we see that it is profitable to sell marginal capacity immediately if

$$
c^{-}>\sum_{t=0}^{T(Q)-1} \alpha^{t} \partial \pi\left(\beta^{t} Q\right) \beta^{t}+\alpha^{T(Q)} \beta^{T(Q)} c^{+}
$$

This inequality should be interpreted as follows: $c^{-}$is greater than any element on the right-hand side of (ii). If the converse of inequality (ii) holds, then marginal capacity should be retained. It follows that we are indifferent between the two alter- 
natives provided

$$
c^{-} \in \sum_{t=0}^{T(Q)-1} \alpha^{t} \partial \pi\left(\beta^{t} Q\right) \beta^{t}+\alpha^{T(Q)} \beta^{T(Q)} c^{+}
$$

To guarantee the existence of a solution $\bar{Q}$ to (iii) observe that the right-hand side is a monotone decreasing function of $Q$. Consequently the two conditions $c^{-} \leqslant c^{+}$and $\pi_{+}^{\prime}(\infty)<(1-\alpha \beta) c^{-}$suffice to guarantee that (iii) has a solution.

The condition $c^{-} \leqslant c^{+}$has already been imposed, and the inequalities $\pi^{\prime}(\infty) \leqslant 0<c^{-}$implying no loss of realism, would take care of the rest. After this preparation we state forthwith

THEOREM 2. Suppose $c^{-} \in \sum_{t=0}^{T(\bar{Q})-1}(\alpha \beta)^{t} \partial \pi\left(\beta^{t} \bar{Q}\right)+(\alpha \beta)^{T(\bar{Q})} c^{+}$for some $\bar{Q} \geqslant 0$ where

$$
T(\bar{Q})=\min \left\{t: \beta^{t} \bar{Q}<Q^{*}\right\}
$$

If $Q_{0}>\bar{Q}$, then it is optimal to sell $Q_{0}-\bar{Q}$ immediately, not invest before time $T(\bar{Q})$ and then to remain at the level $Q^{*}$ thereafter. If $\bar{Q} \geqslant Q_{0}>Q^{*}$, then no capacity should be sold or bought before time $T\left(Q_{0}\right)$ and one should remain at level $Q^{*}$ from this time on.

Proof. We start by discussing the latter case when $\bar{Q} \geqslant Q_{0}$. Since

$$
\Psi(Q):=\sum_{t=0}^{T(Q)-1}(\alpha \beta)^{t} \partial \pi\left(\beta^{t} Q\right)+(\alpha \beta)^{T(Q)} c^{+}
$$

is a monotone decreasing correspondence and $c^{-} \in \Psi(\bar{Q})$, we get $c^{-} \leqslant \phi$ for some $\phi \in \Psi\left(Q_{0}\right)$.

Thus, it is not optimal to sell immediately. However, in the next period $Q_{1}=$ $\beta Q_{0}<\bar{Q}$.

Thus the same type of situation arises once more and again it is not optimal to sell etc., etc. Now turn to the case $Q_{0}>\bar{Q}$. Then $c^{-} \geqslant \phi$ for some $\phi \in \Psi\left(Q_{0}\right)$. Thus if one faces the choice 'sell now or never,' then one incurs no loss by selling the amount $\bar{Q}_{0}-Q$ immediately.

It remains to be argued that the restricted choice 'sell now or never' entails no loss. To see this, suppose that we sold some capacity first at time $T>0$. Then $T<T\left(Q_{0}\right)$, because evidently $T \geqslant T\left(Q_{0}\right)$ would be suboptimal by theorem 1 . However, by selling at time $T$, the marginal capacity will have produced a marginal revenue

$$
\sum_{t=0}^{T-1}(\alpha \beta)^{t} \pi_{+}^{\prime}\left(\beta^{t} Q_{0}\right)+(\alpha \beta)^{T} c^{-}
$$

which of course is dominated by

$$
c^{-}+\sum_{t=0}^{T-1}(\alpha \beta)^{t} \pi_{-}^{\prime}\left(\beta^{t} Q_{0}\right)
$$

Thus the sale should be advanced to time $t=0$. This completes the proof.

Q.E.D. 


\section{The optimal long-term capacity}

In this section we explore how the optimal steady state capacity $Q^{*}$ depends on the data of the problem. Recall that $Q^{*}$ solves the problem:

$$
\text { maximize } P(Q)=\pi(Q)-(1-\alpha \beta) c^{+} Q \text { subject to } Q \geqslant 0
$$

Since $\pi(0)=0, P(Q)>0$ for some $Q>0$ and $P(\infty) \leqslant 0$, the inclusion

$$
(1-\alpha \beta) c^{+} \in \partial \pi\left(Q^{*}\right)
$$

is both necessary and sufficient for optimality as a result of the concavity of $\pi(Q)$.

The following proposition records that there is reason to increase $Q^{*}$ if capital costs are reduced ( $c^{+}$decreases, $\alpha$ or $\beta$ increases) or if the stochastic environment becomes more favourable. The latter means that the variation is less or that probability is shifted towards high quotas.

PROPOSITION 1. The optimal steady state capacity $Q^{*}$ will increase in any of the cases:

(i) $c^{+}$decreases,

(ii) $\alpha$ or $\beta$ increases,

(iii) $\partial_{Q} \pi\left(Q^{*}, q\right)$ is increasing in $q$ and the distribution $F$ of $q$ is replaced with another distribution $G$ such that $F \geqslant G$ (in this case the distribution $G$ is larger than $F$ in terms of first-order stochastic dominance),

(iv) and finally if $\partial_{Q} \pi\left(Q^{*}, q\right)$ is concave increasing in $q$ and the distribution $F$ of $q$ is replaced by a larger distribution $G$ in terms of second-order stochastic dominance.

Proof. In case (i) and (ii) the left-hand side of (6) will decrease. Recall that $\partial \pi\left(Q^{*}\right)$ is monotone decreasing. Thus, $Q^{*}$ must be increased to restore the inclusion (6).

In case (ii) and (iii) the right-hand side of (6) will increase and $Q^{*}$ must move in the same direction to maintain (6).

\section{Remark}

The case (iv) prompts the following observation. Suppose that $G$ is a degenerate distribution concentrated at the mean of $F$. Then $G$ dominates $F$ in the mean preserving second-order sense. Thus if $\partial_{Q} \pi\left(Q^{*}, q\right)$ is concave in $q$, the optimal capacity under certainty is greater than under uncertainty. This tells that one should carefully consider the effects of random variation before deciding upon the catch capacity. The actual importance of uncertainty will of course depend on the data of the actual problem at hand. We shall return to this issue later on. We now turn to examples. These will all be of the type

$$
\pi(Q, q)=u(\min (Q, q))
$$

where $u$ is some strictly increasing concave function. Note that with this specification $\pi(Q, q)$ is concave and increasing. Consequently, replacing the random quota $q$ with its mean, causes $Q^{*}$ to be overestimated by proposition 1 .

Under specification (8),

$$
\pi(Q)=\int_{0}^{Q} u(q) F(\mathrm{~d} q)+u(Q)(1-F(Q)),
$$


and

$$
\pi^{\prime}(Q)=u^{\prime}(Q)(1-F(Q))
$$

provided $Q$ is a continuity point of $F$.

Thus at such points (6) becomes

$$
Q^{*}=V\left((1-\alpha \beta) c^{+}\right)
$$

where $V$ is the inverse of $u^{\prime}(Q)(1-F(Q))$, i.e.

$$
V(y)=\sup \left\{Q: u^{\prime}(Q)(1-F(Q)) \geqslant y\right\}
$$

for y $\in\left[0, u^{\prime}(0)\right]$.

The case $u(Q)=\gamma Q+\delta$, with $\gamma>0$ is interesting. (Note that $\gamma \geqslant(1-\alpha \beta) c^{+}$is a minimal requirement to operate the fishery.)

Then

$$
V(y)=\sup \{Q: F(Q) \leqslant 1-y / \gamma\}
$$

for $y \in[0, \gamma]$.

In particular, if $F$ is invertible,

$$
V(y)=F^{-1}(1-y / \gamma)
$$

We now give two specific examples applying (10).

\section{Examples}

(i) Suppose $q$ is exponentially distributed with parameter $\mu$ so that

$$
F(Q)=1-\exp (-Q / \mu), F^{-1}(\omega)=-\mu \ln (1-\omega)
$$

and therefore

$$
Q^{*}=-\mu \ln \left(\frac{(1-\alpha \beta) c^{+}}{\gamma}\right)
$$

In this case both the mean and the standard deviation of the quota equal $\mu$. Increasing $\mu$ implies that the distribution $F$ becomes larger in first-order sense (and also in the second order). Proposition 1(iii) predicts that in this case $Q^{*}$ will increase. This is confirmed by formula (11).

(ii) Suppose now that $q$ is uniformly distributed on the interval $[a, b]$. Then

$$
Q^{*}=a+\left(1-\frac{(1-\alpha \beta) c^{+}}{\gamma}\right)(b-a)
$$

Observe that if the fishery is very profitable in the sense that the marginal cost of investment $(1-\alpha \beta) c^{+}$is much smaller than marginal revenue $\gamma$, then $Q^{*}$ is close to the maximal quota $b$. By contrast if $(1-\alpha \beta) c^{+}$is close to $\gamma$, then $Q^{*}$ is close to the minimal quota $a$. Hence, in any case one could incur significant loss by selecting the capacity $(a+b) / 2$ representing the mean quota.

\section{An application}

In this section we put the theoretical results of the preceding sections to work on a practical instance of the problem. The issue at hand is to decide on the optimal catch capacity of the Norwegian purse-seiner fleet. The capacity of this fleet could 
potentially be constrained during the capelin fisheries in the Barents Sea. These fisheries are managed by Norway and USSR on a constant escapement basis. Norway takes $60 \%$ of the catch. Mainly the mature part of the stock is exploited, and after spawning the parent stock dies.

Thus, it is rather well adapted to the real situation to conceive of the quotas as being independent and identically distributed.

- We assume that a vessel

- can catch approximately 25,000 tons of capelin at the average price of $550 \mathrm{NOK} /$ ton,

- is halfway depreciated after 15 years, i.e. $\beta^{15}=1 / 2$ so that $\beta=0.9548$,

$-\operatorname{costs} c^{+}=30$ million NOK,

- faces the variable costs 2.3 million NOK, comprising 1 million for fixed expenses and the rest for wages.

Finally, let the interest rate $r=0.07$ p.a. Then $\alpha \beta=(1 / 1+r) \beta=0.89$ and the left-hand side of (6) equals 3.23 million NOK. The revenue created by an addtional vessel is

25,000 tons $\times 550 \mathrm{NOK} /$ ton $-2 \cdot 3$ million $\mathrm{NOK}=11.45$ million $\mathrm{NOK}$

if it is fully occupied throughout the fishery.

Now (6) reads

$$
3 \cdot 23=11 \cdot 45\left(1-\mathrm{F}\left(Q^{*}\right)\right)
$$

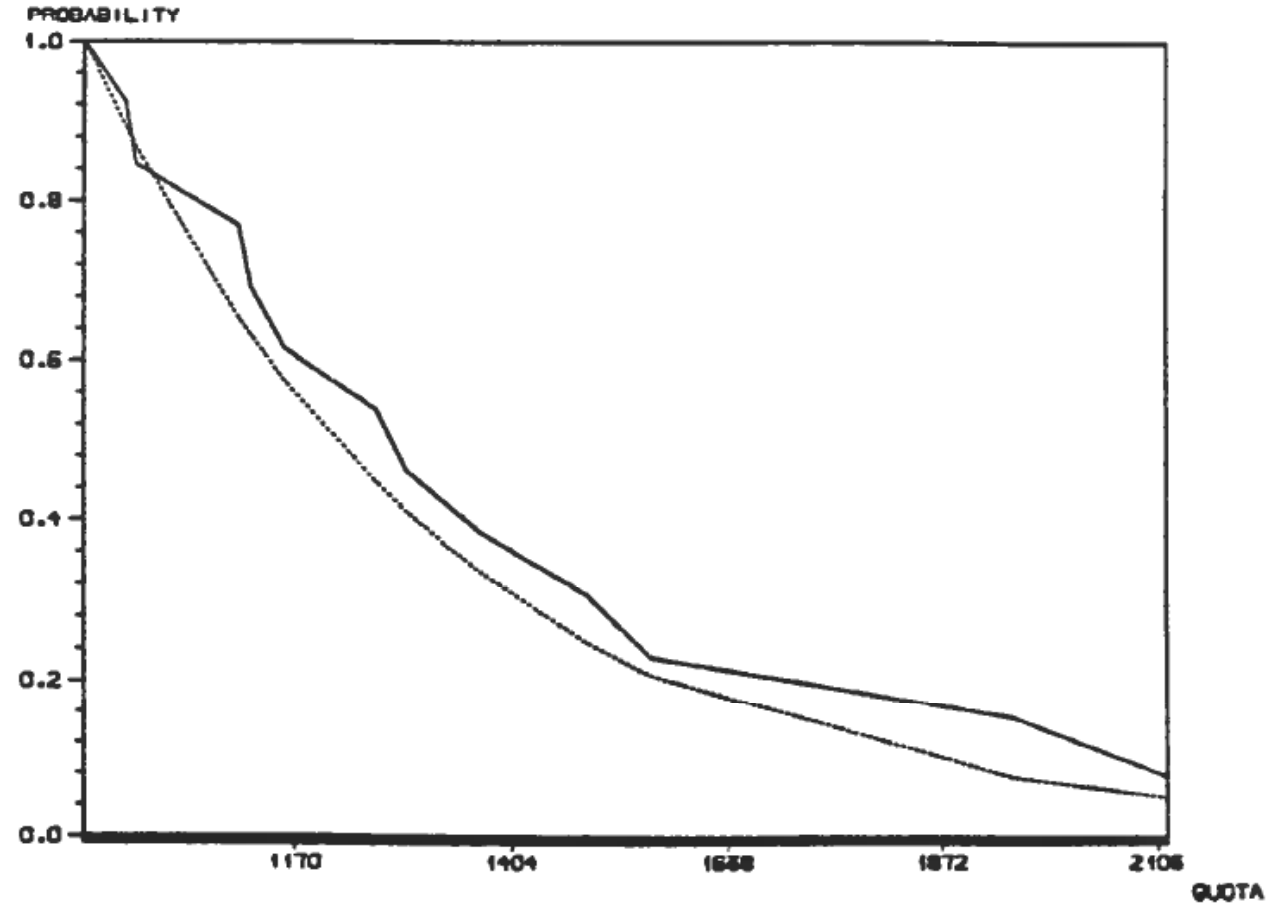

Figure 2. The broken line approximates the empirical distribution $P(Q>$ Quota) in the years 1971-1983. The dotted line is the exponential distribution

$$
P(Q>\text { Quota })=e^{-(\text {Quota-942)/391 }} \text {. }
$$


Hence $P\left(Q>Q^{*}\right)=0.28$. If we identify the unknown theoretical distribution of the quotas with the empirical one as observed in the years 1971-1983 and displayed in Figure 2, we find that $Q^{*}$ is close to $1,500,000$ tons. This quantity corresponds to 60 vessels. Recall that we have ignored possible recourse actions to be implemented if the quota is high. For this reason we have produced an overestimate. We also think that the figures of fixed and variable costs employed above are very moderate. Both of these reservations would tend to lower the size of the optimal fleet.

At any rate the fleet in 1982 comprised more than 130 vessels. Thus capacity is in excess supply and less than half the fleet should remain permanently within the industry. We acknowledge that the existing fleet is by no means a homogeneous population. The economics of scale and technology indicate, however, that small and old vessels ought to leave first. We believe that the necessary restructuring of the industry could make a great leap forward by allowing a free domestic market for licenses possibly constrained by objectives on regional development. Every transaction of licenses should imply an unmodified transfer of a share of the Norwegian quota from seller to buyer. At present the market is not functioning properly, the reason being that by adding another license to a vessel, one gets only a dwindling fraction of the corresponding share. Thus the willingness to pay for an additional license does not match the price demanded by the seller. Consequently few transations take place.

Since capacity is excessive, we now address the question of how far the fleet should be reduced immediately. Towards this analysis we assume that

- the resale value of a vessel $c^{-}=2 / 3 c^{+}=20$ million NOK,

- the marginal revenue $\partial \pi\left(\beta^{t} \bar{Q}\right)$ mentioned in theorem 2 equals approximately $k=1.1$ million NOK for $t=0, \ldots, T(\bar{Q})-1$

$$
\text { (recall that } \partial \pi\left(\beta^{t} \bar{Q}\right) \leqslant \partial \pi\left(Q^{*}\right)=3 \cdot 23 \text { million NOK). }
$$

Then

$$
c^{-}=\frac{1-(\alpha \beta)^{T(\bar{Q})}}{1-\alpha \beta} k+(\alpha \beta)^{T(\bar{Q})} c^{+}
$$

so that $\bar{T}(Q) \approx 6$ years and $\bar{Q} \approx 80$ vessels.

This means that the existing fleet should be reduced by resale rather quickly to about 80 vessels. Thereafter older and smaller vessels should wear out until one reaches the goal level of 60 vessels.

Note that in the case where the quota is exponentially distributed (see Figure 2), i.e.

$$
P(\text { Quota }>Q)=e^{-(Q-a) / b},
$$

then the last part of our argument could easily be made more precise. Indeed, with $u(Q)=\gamma Q+\delta$,

$$
\partial \pi\left(\beta^{t} \bar{Q}\right)=\gamma P\left(\text { Quota }>\beta^{t} \bar{Q}\right)
$$

so that

$$
\sum_{t=0}^{T(\bar{Q})-1}(\alpha \beta)^{t} \partial \pi\left(\beta^{t} \bar{Q}\right)=\gamma \sum_{t=0}^{T(\bar{Q})-1}(\alpha \beta)^{t} e^{-\left(\beta^{t} \bar{Q}-a\right) / b}
$$


The resulting nonlinear generalized equation

$$
c^{-} \in \sum_{t=0}^{T(\bar{Q})-1}(\alpha \beta)^{t} \partial \pi\left(\beta^{t} \bar{Q}\right)+(\alpha \beta)^{T(\bar{Q})} c^{+}
$$

must be solved numerically, however.

\section{Summary}

There is a well-established tradition within practical fisheries management to be predominantly concerned with equilibria of the stocks. In this paper we have taken such an equilibrium, although stochastic in nature, as a datum to which catch capacity should be adapted. We have demonstrated that the path of capacity adjustment is not the same from below the long-term capacity as from above. The reason for this lack of symmetry is found in the irreversible nature of investment. We have also explored how the long-term catch capacity depends on the data of the problem. We have found (see Section 4, example ii) that one cannot in general predict whether capacity will be over-estimated when uncertainty is ignored. A precise answer requires that all parameters, economic and statistical, be considered as a whole.

\section{REFERENCES}

Arrow, K. J., Beckermann, M. J. and Karlin, S. (1958). The optimal expansion of the capacity of a firm, in Studies in the mathematical theory of inventory and production, editors-Arrow, K. J., Karlin, S. and Scarf, H.-Stanford University Press, Stanford, California, 92-105.

Arrow, K. J. (1962). Optimal capital adjustment, in Studies in Applied Probability and Management Science, editors-Arrow, K. J., Karlin, S. and Scarf, H.-Stanford University Press, Stanford, California, 1-17.

BrekKe, K. and Wallace, S. W. (1984). Optimal fleet size when national quotas can be traded, CMI No. 842555-15, 23, Bergen.

Charles, A. (1981). Optimal fisheries investment I: numerical results and comparative dynamics for the deterministic case, Technical Report No. 81-18, University of British Columbia, Institute of Applied Mathematics, Vancouver, Canada.

Clark, C. W., Clarke, F. H. and Munro, G. R. (1979). The optimal exploitation of renewable resource stocks: problems of irreversible investment, Econometrica 47, 25-49.

FLÅM, S. D. and HANNESSON, R. (1983). Uncertain quotas and optimal capacity of the purseseiner fleet, (in Norwegian), Sosialokonomen, 6, 27-32.

FLÅM, S. D. (1981). Steady states in population models with monotone stochastic dynamics, Journal of Mathematical Biology, 13, 87-93.

FLÅM, S. D. and STORøY, S. (1982). Capacity reduction on Norwegian industrial fisheries, Canadian Journal of Fisheries and Aquatic Sciences, 39, 9, 314-1317.

FLÅM, S. D. (1984). Optimal capacity in fisheries: A case where randomness is of minor importance, Annals of Operations Research, 1, 79-83.

Heyman, D. P. and Sobel, M. J. (1984). Stochastic models in Operations Research, Vol. II, McGraw-Hill, New York.

Mirman, L. J. and SPUlBer, D. F. eds. (1982). Essays in the economics of renewable resources, North Holland, New York.

Mirman, L. J. and ZiLCHA, I. (1975). On optimal growth under uncertainty, Journal of Economic Theory, 11.

REED, W. J. (1974). A stochastic model of renewable animal resource, Mathematical Biosciences, 22. 